\title{
Hubot: A Three State Human-Robot Collaborative Framework for Bimanual Surgical Tasks Based on Learned Models
}

\author{
Pierre Berthet-Rayne, Maura Power, Hawkeye King, Guang-Zhong Yang Fellow, IEEE
}

\begin{abstract}
The recent evolution of surgical robots has resolved a number of ergonomic issues associated with conventional minimally invasive surgery (MIS) in terms of aligned visiomotor axes, motion scaling and ergonomics. One of the latest advances is the introduction of human-robot cooperative control combining features such as active constraints, machine learning and automated movements. This paper aims to integrate these techniques into a framework which can be generalized to a wide range of surgical tasks. This paper proposes a system entitled Hubot; a Human-Robot collaborative framework which combines the strengths of the surgeon, the advantages of robotics and learning from demonstration into a single system. Hubot was successfully implemented on a Raven II surgical robot and a user study was conducted to evaluate its performance. Both a training and a simulated clinical case were investigated and showed promising results in comparison to fully manual task execution, including reduced completion time, fewer movements for the operator and improved efficiency.
\end{abstract}

\section{INTRODUCTION}

Contemporary master slave robotic interfaces function as a sophisticated extension of the surgeons hands. Technologies such as machine learning and haptic feedback are currently not available in these systems, but there is potential to incorporate further intelligence and shared knowledge between surgeon and robot.

Learning from demonstrations [1] (LfD) is an ever-growing research field that enables a robot to learn from a human based on task demonstrations. This approach has the advantage of being intuitive to the task provider, while also being extremely powerful by allowing for the quick transfer of a complex task to the robot that would otherwise require months of software development [2]. LfD allows the creation of task models that can then be used for robot control or for human skills evaluation [3]. Lfd can also be used to optimize the human input and outperform the human capabilities and performance [4], [5]. In the future, it can be expected that robotic surgery systems using LfD will be able to safely perform surgery autonomously, with accuracy and speed exceeding that of the best surgeons. Current research into autonomous surgical robotics is actively tackling this problem, however there is still a significant amount of work to do before an autonomous robotic surgery system can be approved for real surgery.

Pierre Berthet-Rayne, Maura Power, Hawkeye King and Guang-Zhong Yang are with the Hamlyn Centre for Robotic Surgery, Imperial College London, SW7 2AZ, London, UK (email:ptb14@imperial.ac.uk)
One possible approach towards fully autonomous systems is to augment robotic devices with features such as partially automated movements and active constraints, to aid a human operator with the completion of a surgery. Continuous Hidden Markov Models (CHMM) [6] is one such LfD method that has been proven as a strong candidate for applications with a surgical context. CHMM has been successfully used in human robot collaborative control for catheterisation [7] as well as surgical training [8], [9] and in humanoid robot control [10]. CHMM is a stochastic approach that aims to model the statistical properties of an observed signal.

This work focuses on safe human-robot collaboration integrating several modes of operation, using LfD and haptic guidance, with varying combinations of control assigned to the human and robot depending on the current context of the surgical procedure. This paper proposes a novel three-state Human-Robot cooperative control framework for bimanual surgical tasks: Hubot, combining the situational awareness of the surgeon, the accuracy and intuitive control of a robot, and the ability to learn from human experience using LfD.

Hubot was implemented on a peg transfer training task [11], and a simulated clinical case: a robotic myomectomy with a $\mathrm{CO}_{2}$ laser, a novel surgical technique offering promising patient outcomes [12]. Myomectomy is the removal of fibroids located inside the uterus. Fibroids are benign tumours, varying in sizes and location, known to cause abdominal or back pain combined with bleedings, pregnancy disorders and even infertility [13]. In a study from Vessey et al., fibroids were also found to be the main cause of total hysterectomy [14]. Although fibroids are benign, Buttram and Reiter evaluated to $25 \%$ the amount of women requiring an intervention due to complications [15], in an article cited by Lumsden [13]. Multiple treatment options exist such as medical treatments, embolization, nanoparticles, and surgical interventions. Although Hubot can be applied to multiple clinical cases, robotic myomectomy can benefit from all the features of the collaborative framework such as haptic guidance, automated movements, and in-situ recording.

The paper is organized as follows: Section II describes the methodology including model learning and task execution. The experimental setup for the user studies is presented in Section III, and the results in Section IV. Finally, Section V concludes with the findings of the work and the potential of this system for future human-robot collaboration. 


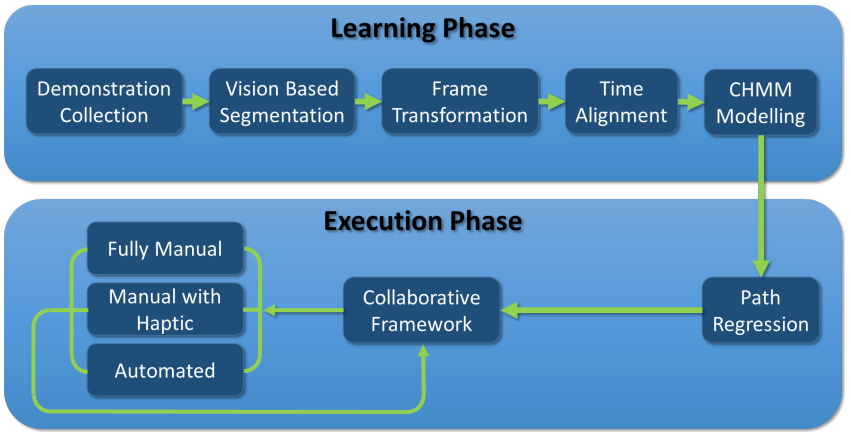

Fig. 1: Hubot's general workflow showing the two main phases. The learning phase creates a model of the surgical task using learning from demonstration, and the execution phase loops between the three states of the collaborative control in real time.

\section{Methodology}

Hubot is a framework allowing a surgeon to work in collaboration with a robot. During a surgical procedure, some motions are more critical than others as they have more potential to cause harm. A tool-tissue or tool-tool interaction, for instance, is critical and therefore should be performed by the surgeon while other types of motions could be automated. Hubot runs in real time and allows for seamless and automatic transitions between three different modes, depending on the current step of the surgical procedure: automated, manual with haptic guidance and finally fully manual. Hubot was implemented on a Raven II surgical robot (Applied Dexterity, Seattle, WA) augmented with a custom absolute controller (available as an open source project) and teleoperated with two Geomagic Touch haptic devices (Geomagic, Morrisville, NC). Figure 1 presents the general workflow of the Hubot collaborative framework, arranged in two main phases: the learning phase and the execution phase.

\section{A. Learning Phase}

1) Task Demonstration: The first step of the workflow is to collect demonstrations of a surgical task. During a demonstration the surgeon performs a task as he/she would during a standard procedure. While performing the task, key information is recorded from the robot and the stereovision system. The recorded robot data is comprised of the absolute Cartesian position of the end effector (3D), the absolute orientation of the end effector as a quaternion (4D) and the absolute grasper position (1D); forming a 16D vector when the two arms' information is combined during bimanual tasks. On the vision side, coloured landmarks are introduced and tracked in 3D in the surgical scene. Two landmarks are used to track the robot's end effectors, and two are attached to the surgical site of interest, forming a 12D vector when the four $3 \mathrm{D}$ points are combined. The recording rate is set to $50 \mathrm{~Hz}$.

2) Automatic Task Segmentation: Once the demonstrations are recorded, the surgical task is segmented into key primitive motions called surgemes [16]. The segmentation is automated and is based on tool-tissue or
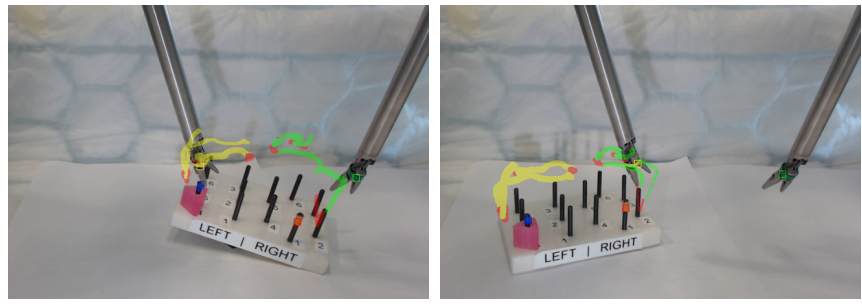

Fig. 2: Hubot adapting to different peg board locations and orientations using visually tracked coloured fiducial markers. The plotted green and yellow trajectories represent the generated "automated" guiding paths, and the "manual with haptic" part is in red (the diameter of the path becomes thinner as the distance from the camera increases).

tool-tool interaction. The presented method uses stereovision to track the 3D position of coloured fiducial markers placed on the two end effectors of the robot and on specific landmarks. This information is then used to measure combined distances between the left and right tool (tool-tool interaction), as well as the left or right tool to the surgical site markers (tool-tissue interaction). Once a distance falls below a chosen safety threshold, an interaction is detected, and the task is segmented. Points below the threshold are set to manual, and the remainder will be classified as automatic. The safety distance is user-adjustable (in $\mathrm{mm}$ ) and ensures that a safety margin to the interaction point will be maintained. To avoid instability due to vibrations during the demonstrations, a hysteresis threshold is used to ensure a more robust segmentation. A complete surgical task will have multiple surgemes which will be automatically segmented. The resulting classification will later be used as a decision parameter to switch between the three modes of the framework.

3) Task Generalization: For a task to be reproduced in a new environment, where the landmarks have changed position, it is necessary to transform the demonstration data, so it becomes landmark position independent. To achieve this, each surgeme is made relative to the desired landmarks. In this context, the following hypothesis is made: Each surgeme is a motion toward a key target point: the coloured landmark position (corresponding to a point of interest) returned by the stereovision system.

During this transformation phase, the robot state and the vision information are combined. The vision information gives the current position of landmarks in the camera frame. The landmarks' position are transformed into the robot space using hand-eye calibration, and are then used as reference points to transform the trajectory of the segment relative to them. This process is repeated for all segments, ensuring that each sub-task is not dependent on absolute position anymore. Once learned task surgemes have been represented relative to landmarks, it is trivial to apply these surgemes to new, relocated landmarks. Figure 2 demonstrates two regressed path for the left (yellow) and right (green) tools. The overlaid path is transformed from the Raven space to 
the camera space, and the resulting points are projected onto the image plane. The parts of the path in red correspond to the manual with haptic guidance mode.

4) Time-Alignment: Each surgeme demonstration is usually of different duration and pace. This is due to natural human variability. In order to perform learning with CHMM, sub-task demonstrations must have the same time duration and time points should correspond between the various demonstrations. Dynamic Time Warping (DTW) [17], [18] is a common approach to time alignment in LfD. For this study, Hierarchical DTW is used as it offers faster computation time and multi-dimensional capabilities [19]. The algorithm is applied to the 16D dataset of the two arms.

5) Task Modelling: CHMM is a doubly-stochastic method of encoding time-dependant or sequential data, making it ideal for demonstration trajectory datasets. A CHMM with $N$ states and $D$ dimensions is represented by:

$$
\lambda=(\pi, A, \mu, \Sigma)
$$

where $\pi$ is the prior probabilities matrix describing the likelihood of starting in each of the $N$ states, $A$ is the state transition matrix describing the likelihood of transitioning from one state to another, $\mu$ is the state emission mean matrix which describes the locations of the centres of the $N$ states in $D$-dimensional space and $\Sigma$ is the state emission covariance matrix which describes the distribution of the $N$ states in $D$-dimensional space. The interdependencies of the sixteen dimensions of the time-aligned task demonstrations are captured and encoded in the trained CHMM, so that a compact representation of the task can be stored for future use and analysis; in this case, the learned CHMM is regressed for control and haptic guidance.

As surgemes are of different length and complexity, the suitable number of states required for modelling are also different. The more complex the sub-task, the more states are required to perform the modelling. An automated brute force algorithm for selection of the number of states was developed. This algorithm creates multiple CHMM models of the same task with a different number of states for each, and automatically selects a good compromise between the minimum number of states required and the highest likelihood of the model. The resulting CHMMs created for each segment are then stored for the execution phase.

\section{B. Execution Phase}

The execution phase uses the models generated in the learning phase to aid with subsequent executions of learned tasks. The first step, is the regression of new trajectories based on the CHMM models, followed by Hubot's main loop execution.

1) Model Regression: As described in section II.A.5 a CHMM contains a mean matrix $\mu$ and a covariance matrix $\Sigma$ describing the distribution (Gaussian) of the data around each mean. Therefore, Gaussian Mixture Regression

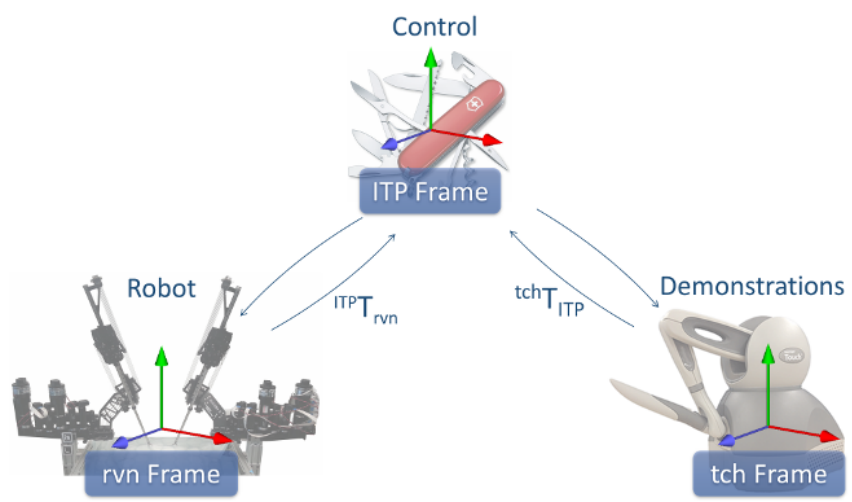

Fig. 3: Frame transformations required to alternate between the three different modes. The demonstrations and haptic rendering are performed in the geomagic touch's frame (tch). The absolute controller (automated mode) is expressed in the ITP frame. The raven position and orientation are expressed in the raven (rvn) frame.

(GMR) [20] is a suitable method to regress a path that will reliably capture the demonstrations. As the CHMM model was created from relative motions, each regressed segment will therefore be relative, and the new configuration of the environment will be required to execute the regressed path with the robot.

2) Hubot: Three State Collaborative Framework: Hubot will now run in a continuous loop, alternating between the three different modes: fully manual mode, manual with haptic guidance and automated. The following paragraphs describe each mode.

\section{(a) Fully Manual Mode:}

In this mode, similar to standard master-slave interfaces, teleoperation of the robot is fully manual and the surgeon has full control. Surgical tasks are performed as normal, with one difference; the motion data of each arm of the robot is monitored and can be recorded in-situ. Fully manual mode is used for surgical sub-tasks that were not previously learned, to take over the automated mode if it needs to be adjusted, or to deal with unexpected conditions. The in-situ recording of the robot motion can be used to replay a task autonomously. This can be particularly useful in cases which require repetitive motions, such as the use of a laser to cut to specific depth. Recorded tasks can also be stored for later use and further improvement of the learned models.

\section{(b) Manual With Haptic Guidance Mode:}

During surgery, if a critical surgeme is detected, Hubot will switch to the manual with haptic guidance mode. In this mode the surgeon has full control over the robot, but with one significant difference: the master interface is augmented with haptic feedback based on the regressed path. Forbidden regions are placed outside of the desired path, therefore guiding the user along the learned trajectory. Forbidden regions were chosen as the most suitable active constraints method as they allow the user to perform the task with a slightly different style from the demonstrations, and compensate for 
small cumulative position errors.

The first step consists of matching the current position of each arm of the robot to the corresponding point on the regressed path. A windowed search algorithm finds the point with the closest Euclidian distance to the current robot position, as shown in equation 2. The search consists of calculating the distance of the current robot position in the raven frame ${ }^{r v n} P$ to all $i$ points of the regressed path ${ }^{r v n} G[i]$ inside the window, and returns the index $i d^{c t}$ of the closest point, corresponding to the current location $c t$ in the path. The window search begins at the current position in the path: $i d^{n}$ (preventing the user from moving backward in the path), to the size of the window $w$ (experimentally set to 30). The metric used is the weighted Euclidean distance between the current robot position $(x, y, z$, grasp $)$ and each point of the regressed path. The grasper angle is given twice the weight of the other elements in the distance calculation, as it is a critical piece of information in the completion of a subtask. The resulting equation is shown below:

$$
i d^{c t}=\underset{i}{\operatorname{argmin}}\left(\operatorname{dist}\left({ }^{r v n} G[i],{ }^{r v n} P\right)\right), i \in\left[i d^{n}, i d^{n+w}\right]
$$

Once the position in the guiding path $i d^{c t}$ is known, the corresponding guiding path point ${ }^{r v n} G[i]$ and current Raven position ${ }^{r v n} P$ are transformed into the ITP frame (Interoperable Teleoperation Protocol [21]) giving ${ }^{I T P} G$ and ${ }^{I T P} P$ respectively. Using the ITP intermediate frame makes the haptic rendering compatible with a range of master interfaces, including the Geomagic Touch. As each arm of the Raven system has its own reference frame, two separate transformations are required as shown in equations 3 and 4. The multiple transformations are shown in figure 3 .

$$
\begin{gathered}
\left\{\begin{array}{l}
I T P_{L} G={ }^{I T P_{L}} T_{r v n_{L}} \cdot{ }^{r v n_{L}} G\left[i d^{c t}\right] \\
I T P_{R} G=I T P_{R} T_{r v n_{R}} \cdot r v n_{R} G\left[i d^{c t}\right]
\end{array}\right. \\
\left\{\begin{array}{l}
I T P_{L} P={ }^{I T P_{L}} T_{r v n_{L}} \cdot r v n_{L} P \\
I T P_{R} P={ }^{I T P_{R}} T_{r v n_{R}} \cdot r v n_{R} P
\end{array}\right.
\end{gathered}
$$

Once ${ }^{I T P} G$ and ${ }^{I T P} P$ are known, the distance vector: ${ }^{I T P} \Delta$ between the two is calculated using equation 5 . This vector represents the distance between the current robot position and the desired position in the regressed path.

$$
\left\{\begin{array}{l}
I T P_{L} \Delta={ }^{I T P_{L}} G-{ }^{I T P_{L}} P \\
I T P_{R} \Delta={ }^{I T P_{R}} G-I T P_{R} P
\end{array}\right.
$$

During teleoperation, users have the possibility to adjust the scale of motion between the master and the slave. Moreover, users will need to clutch when the limit of the master's workspace is reached. Therefore the next step consists of making ${ }^{I T P L} \Delta$ relative to the last clutch position of the master interface: ${ }^{I T P} P_{c t h}$. The haptic target vector: ${ }^{I T P} t g$, expressed in the ITP reference frame is computed using equation 6.

$$
\left\{\begin{aligned}
I T P_{L} t g & ={ }^{I T P_{L}} P_{c t h}+{ }^{I T P_{L}} \Delta \\
I T P_{R} t g & ={ }^{I T P_{R}} P_{c t h}+{ }^{I T P_{R}} \Delta
\end{aligned}\right.
$$

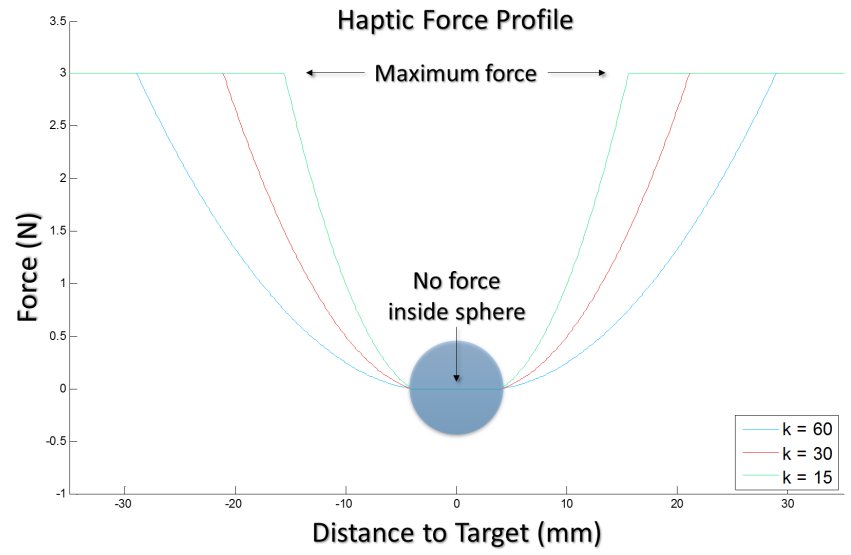

Fig. 4: Plot of the active constraints force profile during the "manual with haptic feedback mode". The force is smoothly rendered to the user to avoid sudden jerky motion. The force profile coefficient $\mathrm{k}$ is used to tune the force profile.

The following step consists of transforming ${ }^{I T P} \mathrm{tg}$ into the corresponding touch reference frame: ${ }^{t c h} t g$ using the transformation matrix: ${ }^{t c h} T_{I T P}$ and scaling it with the user interface scale $U_{s c l}$ as shown in equation 7 .

$$
\left\{\begin{array}{rl}
t c h_{L} & t g=\frac{{ }^{t c h}{ }_{L} T_{I T P_{L}} \cdot{ }^{I T P_{L}} t g}{U_{s c l}} \\
t c h_{R} t g & =\frac{{ }^{t c h_{R}} T_{I T P_{R}} \cdot{ }^{I T P_{R}} t g}{U_{s c l}}
\end{array}\right.
$$

The final step is to render the force: ${ }^{t c h} F$ to the master interface. Forbidden region guidance was implemented in the following manner: the user is allowed to move freely in a sphere around the current point. As there are multiple closely-spaced points along the guiding path, the spheres will intersect, resulting in a tubular shaped trajectory within which the user can move freely. The force is based on the distance of the master interface to the desired target vector. If the distance is below the size of the sphere radius: $S_{r d}$, no force is applied. If it is greater, then force is applied as described in equation 8.

$$
\left\{\begin{array}{l}
{ }^{t c h_{L}} F=\frac{\|\|^{I T P_{L}} \Delta \|-S_{r d}}{k \cdot S_{r d}} \cdot{ }^{t c h} t g: i f\left\|^{I T P_{L}} \Delta\right\|>S_{r d} \\
{ }^{t c h_{R}} F=\frac{\left\|^{I T P_{R}} \Delta\right\|-S_{r d}}{k \cdot S_{r d}} \cdot{ }^{t c h} t g: i f\left\|^{I T P_{R}} \Delta\right\|>S_{r d} \\
\left.t c h_{L \mid R} F=0: i f\left\|^{I T P_{L \mid R}} \Delta\right\| \leq S_{r d}\right)
\end{array}\right.
$$

Finally the force is bounded by a maximum value. The resulting force profile ensures a continuous rendering of forces and a smooth transition when forces start to be applied as shown in figure 4 . The force profile coefficient $k$ was implemented to adjust the shape of the global profile and was experimentally set to 60 , rendering a force strong enough for the user to feel it, but not too strong that it would create sudden kickbacks.

\section{(c) Automated Mode:}

In this mode, the control of the robot is fully automated. This mode is only active when no tool-tool or tool-tissue interaction is detected. When active, the two arms of the robot move along the regressed path learned from previous 
demonstrations. Typically, automated motions are movements to transition between various points of interest in the surgical field to connect two manual-mode surgemes together.

Visual cues in the user interface inform the surgeon when the automated mode is on. It is important to note that at any time the surgeon can take over the automated mode and switch seamlessly to the fully manual mode. The transition from automated to manual with haptic is instantaneous once the robot has reached the end of the automated path. A single guiding path is used for the complete task, whether in manual or automated mode. Therefore, the robot's end effector is correctly placed when switching from automated to manual with haptic. This ensures smooth transitions without sudden changes in applied forces. When transitioning from manual to automated however, the position of the end effector can be slightly different from the desired one. To avoid abrupt motion of the robot, the developed Raven II absolute controller will linearly interpolate the path between its current location and the desired one, resulting in a smooth transition.

\section{EXPERIMENTAL SETUP}

A user study was conducted to evaluate the benefits of Hubot. Although Hubot can generalize learned surgemes to changed environment, for the purpose of the user study the landmarks were attached to a board and an absolute model was created. Therefore, all the participants used the exact same configuration. This choice was made to avoid confounding factors resulting from certain position and orientation being more difficult than others to perform the task, due to vision angle or colliding tool configuration. During the study, 20 subjects, with and without experience in robotic teleoperation or surgery, were recruited. Three of the participants were experienced surgeons. During the study each participant was asked to perform the same two surgical tasks: The first task consisted of a peg transfer task. Each subject was asked to perform four peg transfer alternating between a fully manual task and a collaborative task with Hubot. The second task consisted of performing a simulated robotic myomectomy with a $\mathrm{CO}_{2}$ laser. This task was performed twice by each participant, first in fully manual mode and then with Hubot. The possible learning effect are considered in the discussion of the user study results.

\section{A. Setup}

During the study, the participants used two Geomagic Touch devices to control the Raven II robot. A custom handle was designed to replace the standard Geomagic stylus. This handle, called the HIG-Touch (Handheld Intuitive Grasper for Geomagic Touch) mimics the grasper movements resulting in a more intuitive control of the robot (available as an open source project). A set of three foot pedals was also provided. The users had no direct view of the Raven and the task scene. Instead, a 3D endoscope was installed (Olympus, Tokyo, Japan), and the participants were provided with 3D glasses and a 3D screen showing the robot arms and the surgical scene.
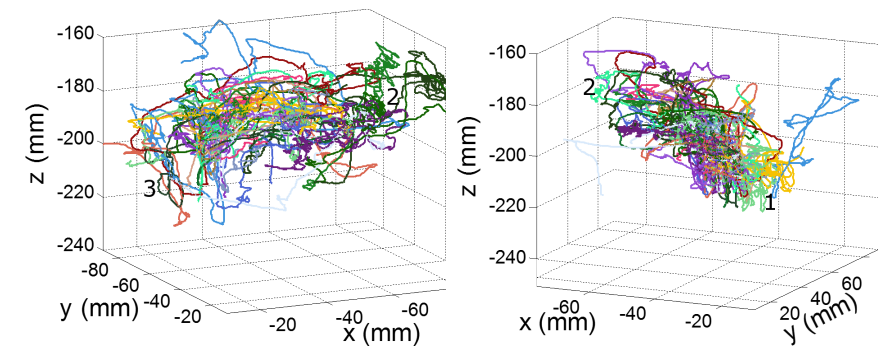

(a) Left tool path (Hubot off)

(b) Right tool path (Hubot off)
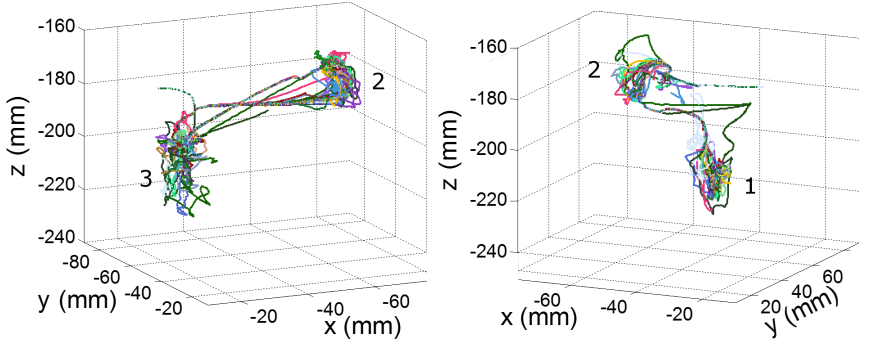

(c) Left tool path (Hubot on)

(d) Right tool path (Hubot on)

Fig. 5: Comparison of the tool path with and without Hubot during the peg transfer task. Each user's path is represented with a different color. The resulting Hubot trajectories are more consistent and show clear motion primitives during the pick-up, transfer and release phases marked as 1, 2 and 3 respectively.

\section{B. Peg Transfer Task}

The peg transfer task is performed using a standard FLS training [11] board. The task consists of picking a triangular piece of rubber from a peg with one tool, transferring it from one tool to the other, and finally replacing it onto another peg on the opposite side. During standard FLS training, this task is performed until all the blocks from one side of the board have been moved to the opposite side and back. During the user study, the task was simplified to have a similar level of difficulty among all the participants. The four peg transfers were performed between the same two pegs, one marked in red and another one marked in blue for visual tracking.

\section{Simulated Robotic Assisted Myomectomy with $\mathrm{CO}_{2}$ Laser}

Out of the 20 participants in the user study, 13 decided to proceed with the simulated clinical case. For the purpose of the user study, the simulated task was composed of the following steps, illustrated in figure 7 . First the user needs to move organs and tissue to access the uterus. The second step consists of performing three passes of $\mathrm{CO}_{2}$ laser to cut through the uterus and access the tumour. The $\mathrm{CO}_{2}$ laser was simulated, for user safety, by a LED mounted on an optic fibre. The fibre was equipped with a pick-up holder allowing the Raven II standard tool to easily grasp it. The third and final step consists of lifting the cut piece of tissue and removing the fibroid with a standard grasper.

\section{RESUlts}

\section{A. Peg Transfer Task}

1) Slave Trajectory Comparison: Figure 5 shows the path followed by the robot during the first manual trial of the peg 
transfer task (top) and the fourth trial with Hubot (bottom). Each of the 20 participants were assigned a unique colour in the plot. The plotted trajectories clearly show the difference between the two modes. The manual mode data is chaotic, and it is difficult to distinguish any pattern. The Hubot data however visibly shows a movement pattern during the grasping of the block (1), the exchange point (2) and the release location (3).

2) Total Time to Complete the Task: Figure 6a shows the distribution of the total time to complete the task for the 20 participants in a box plot form. During the first manual trial, each participant had a different technique as well as a different level of expertise. Therefore, the resulting box plot shows a large variation, with large error bars and an average time to complete the task of approximately 100 seconds. During the second trial with the aid of Hubot however, the distribution of the data is much less, with smaller error bars showing a similar overall performance amongst the participants. The average time was reduced to 56 seconds, dividing almost by two the average time of the first trial. During the third trial, the data is again largely distributed with an average time of 75 seconds, but the overall performance is significantly better than the first trial as the variation is smaller and the completion time was reduced by $25 \%$.

This data shows that the participants are learning during each step. This was also observed while conducting the study, as most of the users with no experience tried to replicate the movements they had just performed while collaborating with Hubot. Finally during the fourth trial with Hubot, the average time decreased to 42.5 seconds with a narrowly distributed data showing a general improvement across all the participants.

3) Master Total Travelled Distance: The total distance travelled by the master interface (figure $6 \mathrm{~b}$ and $6 \mathrm{c}$ ) represents the amount of motion the participants had to perform with the master input device during the task. For the first trial in manual mode, the distribution of the data is large with an average distance of $58 \mathrm{~cm}$ for the left hand and $49 \mathrm{~cm}$ for the right one. During the second trial with Hubot, the travelled distance is significantly reduced, with an average path length of $20.0 \mathrm{~cm}$ for the left and $14.6 \mathrm{~cm}$ for the right, representing an important reduction of the required amount of motion by $65 \%$ for the left hand and $70 \%$ for the right hand. In the third trial, the distribution of the data becomes large again, with an average distance of $57.6 \mathrm{~cm}$ for the left and $43.2 \mathrm{~cm}$ for the right. There is no significant improvement from the first trial, but the distribution is slightly reduced, showing a small general improvement from the first manual trial. Finally, in the fourth trial with Hubot the average distance on the left is reduced to $17.4 \mathrm{~cm}$ and $12.8 \mathrm{~cm}$ for the right, representing a substantial reduction of $70 \%$ for the left and $74 \%$ for the right from the first trial. This significant improvement is due to the robot performing part of the task, but also to the active constraints, which prevent the user from moving unnecessarily during the task.
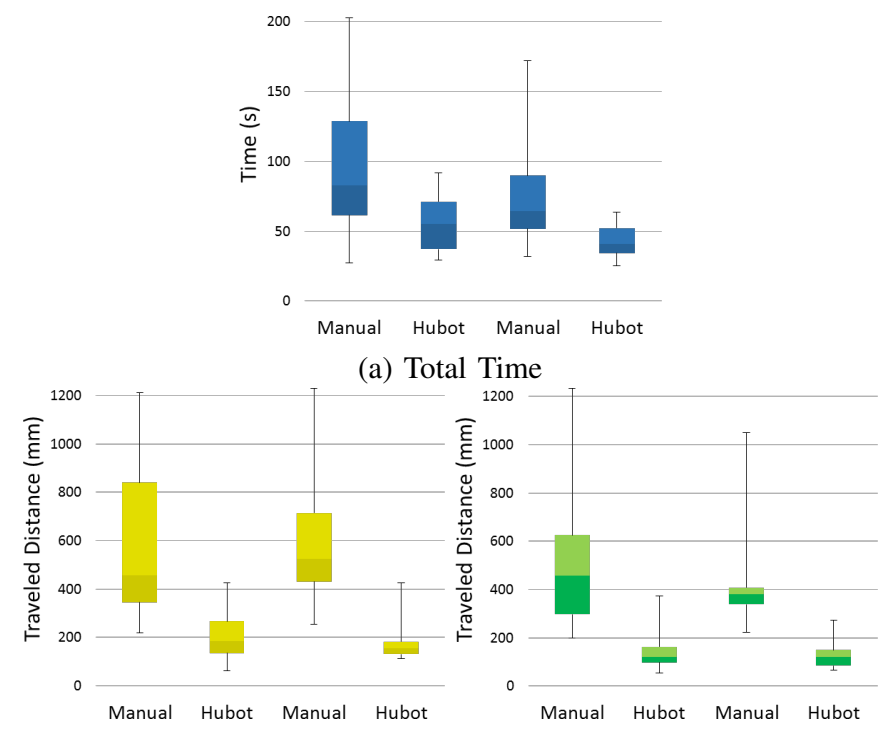

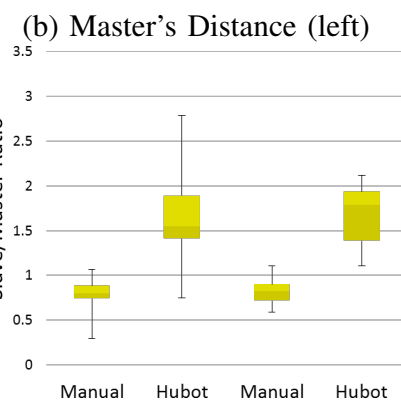

(d) Efficiency (left) (c) Master's Distance (right)

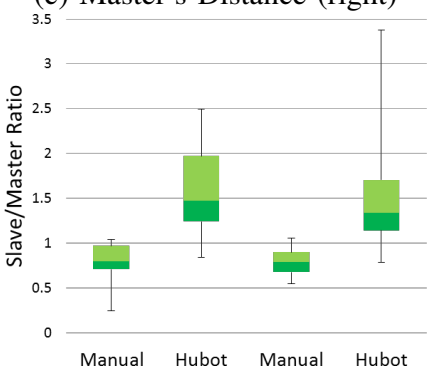

(e) Efficiency (right)
Fig. 6: Comparative results of the peg transfer user study.

4) Operation Efficiency: The operation efficiency is the ratio of the slave-travelled distance over the master-travelled distance, representing how efficient the user's motions were relative to the length of the task being performed by the robot. It is important to note that all participants used the same teleoperation scaling factor during the study.

As shown in figure $6 \mathrm{~d}$ and $6 \mathrm{e}$, during the four trials, a general improvement in the operation efficiency is revealed using Hubot for both the left and right hand. This trend was expected since during automated motion the user is not required to move the master interface, and when Hubot switches to the manual with haptic mode the tool is already positioned and oriented, avoiding the need to clutch to reposition the master interface. In addition, active constraints limited unnecessary movements.

\section{B. Robotic Myomectomy}

1) Total Time to Complete the Task: The total time to complete the task results are coherent with the peg transfer results. As shown in Figure 8a, the total time to perform the task is reduced from an average time of 240.6 seconds to 186.2 seconds, representing a reduction by $22.6 \%$. The distribution of the data is also slightly narrower, showing a general improvement of the participants. 

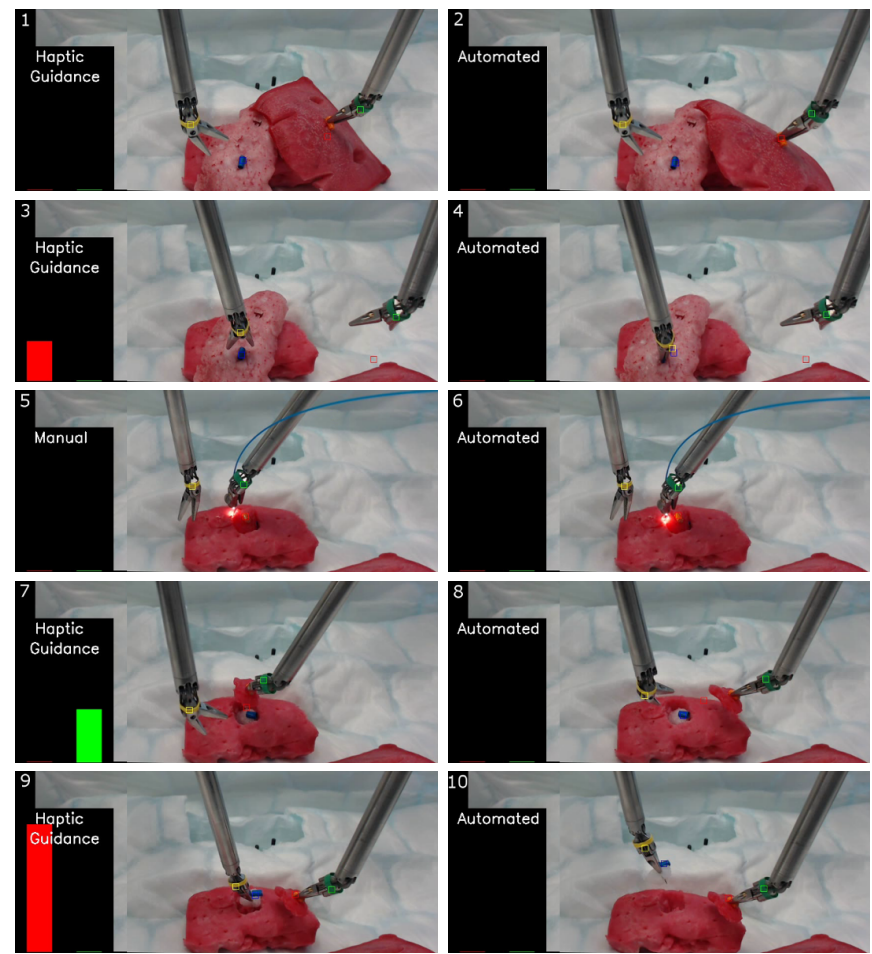

Fig. 7: Time lapse of the simulated robotic myomectomy task segmented into 10 surgemes. The live state of the robot is displayed at the top of the rectangular black box. The red and green bar graph represents the norm of the force vector being rendered to the left and right master interface respectively.

2) Master Total Travelled Distance: As in the previous task, the total distance travelled by the master interface (figure $8 \mathrm{~b}$ and $8 \mathrm{c}$ ) is significantly reduced on both the left and right hands. During the first manual trial, the average travelled distance is $116.4 \mathrm{~cm}$ for the left and $136.2 \mathrm{~cm}$ for the right. During the trial in collaboration with Hubot, the average distance is $55.5 \mathrm{~cm}$ for the left and $62.3 \mathrm{~cm}$ for the right, representing an important path length reduction of $52 \%$ for the left and $54 \%$ for the right hand.

3) Operation Efficiency: The operation efficiency (fig 8d and 8e) during the clinical case is also improved using Hubot for both hands. This shows that even during a complex task, Hubot can benefit the user by increasing the efficiency.

\section{CONCLUSION}

\section{A. Discussion and Future Work}

The presented method was applied to identical tasks during the learning and execution phases. Although Hubot can adapt to new landmark positions, the current implementation cannot compensate for different sizes of landmarks such as a smaller peg board for instance. This limitation could however be improved by scaling the CHMM model. Scaling a CHMM consists of warping the covariance matrix, so the regressed path will correspond to the new environment. In future implementation, this scaling step would use the stereovision system to detect the position of landmarks in the new environment

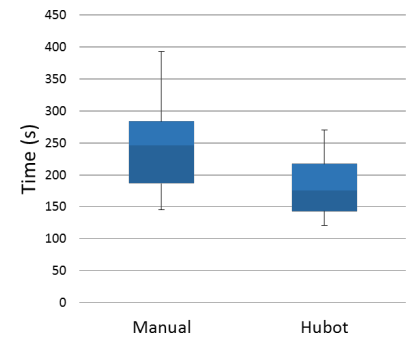

(a) Total Time

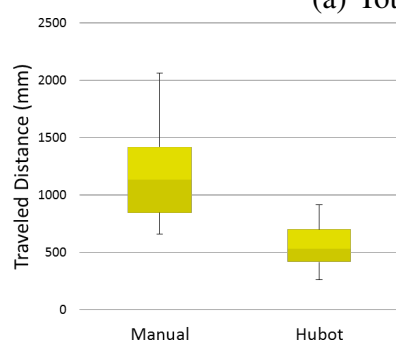

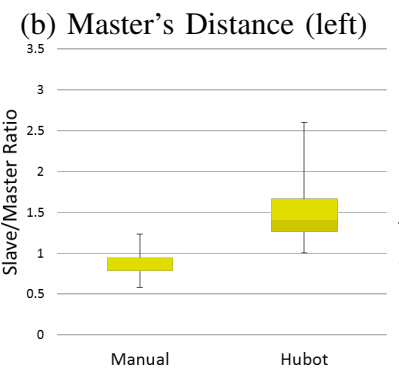

(d) Efficiency (left)

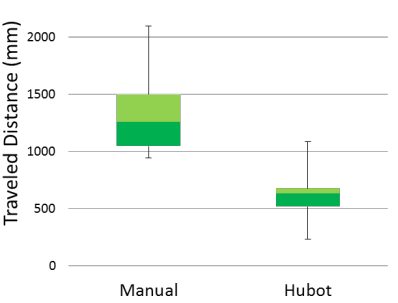

(c) Master's Distance (right)

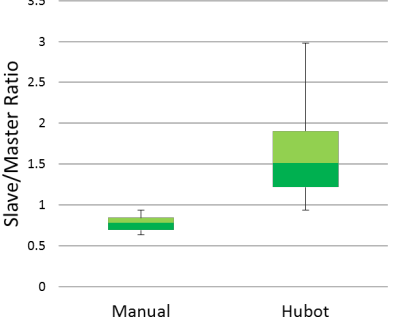

(e) Efficiency (right)
Fig. 8: Results of the simulated myomectomy user study.

and compute the corresponding scaling factor. This approach would result in a more polyvalent implementation of Hubot that could adapt to multiple conditions.

The current implementation of Hubot uses the location of the tools inside the regressed path to detect the current movement performed by the user. CHMM itself offers the potential to estimate the current user's movement and evaluate future intentions using probabilities. These two features combined could result in more robust detections of manual to automated transitions.

The current shape of the forbidden region is constant along the regressed path. The covariance matrix of the CHMM gives information on the variance of the data, and this information can be used to vary the diameter of the allowed region. A large variance indicates that the demonstrations were performed roughly at this specific stage during each trial, whereas a small variance indicates that the user performed consistently accurate motions. This information, combined with the haptic rendering, would result in the growing or shrinking of the forbidden region, respectively allowing or restricting the user movements. If implemented, this new feature would require the generation of a $3 \mathrm{D}$ ellipses around each regressed point rather than a sphere. A simpler version was implemented using rectangular boxes, but was not tested during the user study.

As an example of failure during the peg transfer user study, 
some users incompletely grasped the triangular block and started the lift motion with the block still on the peg. Hubot continued the subsequent mode, and did not allow the user to return to the previous phase unless to switch to fully manual mode. Future implementation of Hubot could use vision to ensure the correct grasp, and offer the possibility to go back to the previous phase.

A comparative study of subjects performing the same tasks, but divided in two separate groups, one performing the task in manual mode only, and the other one performing the task with Hubot, would eventually reveal if Hubot allows subject to learn faster than with the conventional manual method.

\section{B. Conclusion}

This paper contributes to the field of intelligent surgical robots by presenting a polyvalent human-robot collaborative framework called Hubot which can adapt to changing landmark configurations by using stereovision. As Hubot is a high level software, it runs as a layer on top of the robot controller software and does not integrate the kinematic model of the system. Therefore, it can be readily implemented on any existing robot platform such as the da-Vinci surgical robot [22].

This work also introduces a new approach to automated CHMM modelling and automated surgeme segmentation requiring minimal user input during the learning phase. To our knowledge, Hubot is the first framework combining human awareness, robot accuracy, intuitive control, LfD, stereovision and active constraints in a seamless manner toward a simulated clinical case. The Hubot methodology was validated during a user study and showed improvement in the total time to complete the tasks, the total amount of motion required and the efficiency of motion.

Finally Hubot represents a first step toward the complete automation of surgical procedures. Learned surgical task models created for Hubot have the potential to gradually become more automated, until the models are safe enough to become entirely autonomous. As a result, Hubot will allow a smooth and progressive transition from the current teleoperation techniques to fully autonomous surgery.

\section{ACKNOWLEDGEMENTS}

The authors would like to thank the following people for their input: Hedyeh Rafii-Tari (CHMM), Alessandro Vandini (vision), Lin Zhang and Maria Grammatikopoulou (general input), Michael Hughes (simulated laser), Archie HughesHallett (tissue phantoms), Petros Giataganas (pick-up LED holder and 3D prints), Gauthier Gras (hierarchical DTW library) and Burak Temelkuran from OmniGuide (MA, USA) for the $\mathrm{CO}_{2}$ laser application.

\section{REFERENCES}

[1] B. D. Argall, S. Chernova, M. Veloso, and B. Browning, "A survey of robot learning from demonstration," Robotics and Autonomous Systems, vol. 57, no. 5, pp. 469-483, 2009.
[2] S. Schaal, A. Ijspeert, and A. Billard, "Computational approaches to motor learning by imitation.," Philosophical Transactions of the Royal Society of London B: Biological Sciences, vol. 358, no. 1431, pp. 53747, 2003.

[3] P. D. van Hove, G. J. M. Tuijthof, E. G. G. Verdaasdonk, L. P. S. Stassen, and J. Dankelman, "Objective assessment of technical surgical skills.," The British Journal of Surgery, vol. 97, no. 7, pp. 972-87, 2010.

[4] J. van den Berg, S. Miller, D. Duckworth, H. Hu, A. Wan, X.Y. Fu, K. Goldberg, and P. Abbeel, "Superhuman performance of surgical tasks by robots using iterative learning from human-guided demonstrations," in Robotics and Automation (ICRA), 2010 IEEE International Conference on, pp. 2074-2081, May 2010.

[5] A. Coates, P. Abbeel, and A. Y. Ng, "Learning for control from multiple demonstrations," in Proceedings of the 25th International Conference on Machine Learning, ICML '08, (New York, NY, USA), pp. 144-151, ACM, 2008.

[6] L. R. Rabiner, "A Tutorial on Hidden Markov Models and Selected Applictions in Speech Recognition," Proceedings of the IEEE, vol. 77, no. 2, pp. 257-286, 1989.

[7] H. Rafii-Tari, J. Liu, C. J. Payne, C. Bicknell, and G.-Z. Yang, "Hierarchical HMM Based Learning of Endovascular Catheterization," in Int. Conf. on Medical Image Computing and Computer-Assisted Intervention, pp. 496-503, 2014.

[8] M. Power, H. Rafii-Tari, C. Bergeles, V. Vitiello, and G.-Z. Yang, "A Cooperative Control Framework For Haptic Guidance Of Bimanual Surgical Tasks Based On Learning From Demonstration," in IEEE Int. Conf. on Robotics and Automation, pp. 5330-5337, 2015.

[9] N. Padoy and G. D. Hager, "Human-Machine Collaborative surgery using learned models," in IEEE Int. Conf. on Robotics and Automation, pp. 5285-5292, 2011.

[10] A. Billard, S. Calinon, and F. Guenter, "Discriminative and Adaptive Imitation in Uni-Manual and Bi-Manual Tasks," Robotics and Autonomous Systems, vol. 54, no. 5, pp. 370-284, 2006.

[11] SAGES, "Fundamentals of laparoscopic surgery, the definitive laparoscopic skills enhancement and assessment module."

[12] S. E. Barton and A. R. Gargiulo, "Robot-assisted laparoscopic myomectomy and adenomyomectomy with a flexible CO2 laser device," Journal of Robotic Surgery, vol. 7, pp. 157-162, 2013.

[13] M. A. Lumsden, "Embolization versus myomectomy versus hysterectomy: which is best, when?," Human Reproduction, vol. 17, no. 2, pp. 253-259, 2002.

[14] M. P. Vessey, L. Villard-Mackintosh, K. McPherson, A. Coulter, and D. Yeates, "The epidemiology of hysterectomy: findings in a large cohort study.," British Journal of Obstetrics and Gynaecology, vol. 99, pp. 402-407, 1992.

[15] V. Buttram Jr and R. Reiter, "Uterine leiomyomata: etiology, symptomatology, and management," Fertility and Sterility, vol. 36, no. 4, p. $433,1981$.

[16] B. Varadarajan, C. Reiley, H. Lin, S. Khudanpur, and G. Hager, "Data-derived models for segmentation with application to surgical assessment and training.," in Int. Conf. on Medical Image Computing and Computer-Assisted Intervention, pp. 426-34, 2009.

[17] H. Sakoe and S. Chiba, "Dynamic Programming Algorithm Optimization for Spoken Word Recognition," IEEE Transactions on Acoustics, Speech, and Signal Processing, vol. ASSP-26, no. 1, pp. 43-49, 1978.

[18] M. Müller, "Chapter 4: Dynamic Time Warping," in Information Retrieval for Music and Motion, pp. 69-84, Springer, 2007.

[19] G. Gras, V. Vitiello, and G.-Z. Yang, "Cooperative Control of a Compliant Manipulator for Robotic-Assisted Physiotherapy," in IEEE Int. Conference on Robotics and Automation, pp. 339-346, 2014.

[20] S. Calinon, F. Guenter, and A. Billard, "On learning, representing, and generalizing a task in a humanoid robot," IEEE Transactions on Systems, Man, and Cybernetics, Part B: Cybernetics, vol. 37, no. 2, pp. 286-98, 2007.

[21] H. H. King, B. Hannaford, K. W. Kwok, G.-Z. Yang, P. Griffiths, A. Okamura, I. Farkhatdinov, J. H. Ryu, G. Sankaranarayanan, V. Arikatla, K. Tadano, K. Kawashima, A. Peer, T. Schauß, M. Buss, L. Miller, D. Glozman, J. Rosen, and T. Low, "Plugfest 2009: Global interoperability in telerobotics and telemedicine," in IEEE Int. Conference on Robotics and Automation, pp. 1733-1738, 2010.

[22] P. Kazanzides, Z. Chen, A. Deguet, G. S. Fischer, R. H. Taylor, and S. P. Dimaio, "An Open-Source Research Kit for the da Vinci Surgical System," in IEEE Int. Conference on Robotics and Automation, pp. 6434-6439, 2014 